\title{
Electric dipole moments of charged leptons from their Majorana-type Yukawa couplings
}

\author{
Yi Liad 1 \\ Department of Physics, Nankai University, Tianjin 300071, China
}

\begin{abstract}
The electric dipole moments (EDMs) of charged leptons are significantly suppressed in standard model. It has been found previously that they are even more severely suppressed in seesaw type models by powers of tiny neutrino masses as far as a leptonic CP source is concerned. We investigate whether a Majorana-type Yukawa coupling between charged leptons and a doubly charged scalar can contribute significantly to their EDMs. An observable EDM would then help unravel the Majorana nature of neutrinos by a lepton number conserving quantity. We find that the EDMs are indeed parametrically large, of the form $d_{\alpha} \propto e m_{\alpha}\left(m_{\beta}^{2}-m_{\gamma}^{2}\right) / m^{4}$ up to logarithms, where $m_{\alpha}$ and $m$ are respectively the masses of charged leptons and the scalar. And they satisfy a sum rule to good precision, $d_{e} / m_{e}+d_{\mu} / m_{\mu}+d_{\tau} / m_{\tau}=0$. With the most stringent constraints from lepton flavor violating transitions taken into account, their values are still much larger than the mentioned previous results. Unfortunately, even in the most optimistic case the electron EDM is about three orders of magnitude below the foreseeable experimental sensitivity.
\end{abstract}

PACS: 13.40.Em, 14.60.-z, 12.60.Fr, 14.60.Pq

Keywords: electric dipole moment, Majorana neutrino, doubly charged scalar

${ }^{1}$ liaoy@nankai.edu.cn 


\section{Introduction}

The CP violation through a Dirac phase in the CKM matrix of weak interactions has been well tested in the flavored systems of hadrons. It is generally believed however that this cannot be the unique or even the dominant source of $\mathrm{CP}$ violation because of the observed large baryon number asymmetry in our universe (BAU). One of attractive solutions to BAU is offered by the mechanism of leptogenesis [1] in which the lepton number asymmetry is first generated through $\mathrm{CP}$ violation in the lepton sector and then converts partly into BAU via sphaleron effects [2].

Our current information on the leptonic mixing matrix comes dominantly from experiments of neutrino oscillations [3]. The matrix is CKM-like involving a single Dirac phase if neutrinos are Dirac particles, but can contain additional two $\mathrm{CP}$ phases if neutrinos are of Majorana nature. The oscillations are blind to the latter Majorana phases while their sensitivity to the Dirac phase is seriously diminished by a very small, if not vanishing, mixing angle out of three. This leaves $\mathrm{CP}$ violation in the lepton sector largely untested so far except perhaps for the experiment of neutrinoless double beta $(0 v 2 \beta)$ decays which can be sensitive to $\mathrm{CP}$ phases but whose status is under debate.

Nevertheless, there is another physical observable, the electric dipole moment (EDM), that can provide an independent probe to $\mathrm{CP}$ violation. The current experimental limits on the EDMs of the mercury atom [4], neutron [5], electron [6] and muon [7] are already very impressive, and further improvements are expected to take place in the near future [8]. These EDMs can in principle be induced by the Dirac phase in the CKM matrix of standard model (SM). However, it was known long ago that the electric and chromoelectric dipole moments of quarks vanish to the two-loop order [9]. This was interpreted as a joint result of two features in SM [10], namely the unitarity of the CKM matrix and the purely left-handed chirality of the charged current, and relaxation of any of them would yield quark EDMs at two loops [11]. The lepton EDMs then become extremely small in SM as they are first induced at four loops [12]. This makes them a potentially ideal place to search for $\mathrm{CP}$ violation in the lepton sector.

If neutrinos are Dirac particles, the EDMs of charged leptons will be hopelessly tiny. The case of quarks in SM repeats in the lepton sector in an even worse manner since neutrinos can be considered degenerate to very good precision at the weak scale, in which case there is effectively no $\mathrm{CP}$ violation in the lepton sector. But the situation could be different when neutrinos are Majorana particles because of peculiarities with Majorana CP phases [13]. Since Majorana phases dominate over the Dirac phase in this case, an observable lepton's EDM would not only discover $\mathrm{CP}$ violation in the lepton sector but also expose the Majorana nature of neutrinos by a lepton number conserving quantity in sharp contrast to $0 v 2 \beta$ decays. Indeed, as pointed out in [14], there is a topologically new type of two-loop Feynman diagrams when neutrinos are Majorana particles that can contribute to the charged lepton EDMs. But it was found subsequently that this type of contribution is always severely suppressed by neutrino masses from virtual loops whether one works in the standard type I seesaw model [15] or one augmented with an additional Higgs doublet [16], or in type II seesaw [17]. The obtained numbers are actually even smaller than the four-loop result due to the CKM phase, and thus 
would not be observable in any foreseeable experiments.

When neutrinos are Majorana particles, the lepton number may be violated either by a bare Majorana-type mass of heavy neutrinos that are singlets of SM, or by some other fields that are active in SM and couple in particular to leptons. The physics at low energies is much richer in the latter case. And the simplest choice would be to add a scalar triplet as in the type II seesaw model [18]. We are thus motivated to consider the most general Majorana-type Yukawa couplings of charged leptons to a doubly charged scalar. These couplings could arise as, but are not restricted to, part of interactions as in type II seesaw or a larger extension of SM. The $\mathrm{CP}$ violation encoded in the couplings can induce EDMs to charged leptons, and we find that this contribution is indeed parametrically large. Besides the product of Yukawa couplings and a lepton mass factor for chirality flip, the EDM is suppressed by charged lepton masses squared over four powers of the scalar mass and is partly enhanced by a logarithm. In particular, it incurs no suppression by neutrino masses since no neutrinos appear in virtual loops. This is the largest term in lepton EDMs, to our knowledge, coming from a flavor dependent CP source.

The paper is organized as follows. In the next section we describe the Majorana-type Yukawa couplings between the charged leptons and doubly charged scalars, and count the number of independent physical parameters. The two-loop diagrams for EDMs are then evaluated analytically in section 3, and a sum rule is found. Using the most stringent constraints from lepton flavor violating decays we estimate in section 4 the largest allowed values for the EDMs. We summarize and conclude in the last section.

\section{Majorana-type Yukawa couplings}

The relevant interactions for our study are the Majorana-type Yukawa couplings

$$
\mathscr{L}_{\text {Yuk }}=\ell^{T} b \mathscr{C} P_{L} \ell \xi^{++}+\bar{\ell} b^{\dagger} \mathscr{C} P_{R} \bar{\ell}^{T} \xi^{--}
$$

and the standard QED

$$
\mathscr{L}_{\mathrm{QED}}=-e A^{\mu} \bar{\ell} \gamma_{\mu} \ell+2 i e A^{\mu}\left(\xi^{--} \partial_{\mu} \xi^{++}-\xi^{++} \partial_{\mu} \xi^{--}\right)
$$

Here $\ell, \xi^{ \pm \pm}$and $A_{\mu}$ are respectively the charged lepton, doubly charged scalar and electromagnetic fields, and $e$ is the electromagnetic coupling. We use Greek letters to denote the three charged leptons. $\mathscr{C}=i \gamma^{0} \gamma^{2}$ is the matrix employed in charge conjugation and $P_{R, L}=\frac{1}{2}\left(1 \pm \gamma_{5}\right)$ are chiral projectors.

The Yukawa coupling matrix $b$ is symmetric in lepton flavors due to antisymmetry in fermion fields but is otherwise arbitrary. With $n$ flavors of leptons, $b$ has generally $n+\frac{1}{2} n(n-1)$ moduli and $n+\frac{1}{2} n(n-1)$ phases. All moduli are physical parameters. However, not all of the phases are physical. For instance, the phases in the diagonal entries $b_{\alpha \alpha}$ can all be removed by redefining complex fields $\ell_{\alpha}$. After this, there are no more degrees of freedom to rephase fields without reintroducing phases into $b_{\alpha \alpha}$. There are thus only $\frac{1}{2} n(n-1)$ physical phases. They signal $T$ and $C P$ violation as we analyze below. 
When the matrix $b$ is real, we can prescribe the $T$ and $C P$ transformations as $T \xi^{++} T^{-1}=$ $+\xi^{++},(C P) \xi^{++}(C P)^{-1}=-\xi^{--}$so that both are preserved by the above interactions. If $b$ is purely imaginary, we can prescribe in the opposite manner to preserve both $T$ and $C P$, $T \xi^{++} T^{-1}=-\xi^{++},(C P) \xi^{++}(C P)^{-1}=+\xi^{--}$. The latter case can of course be reduced to the former by rephasing the $\xi^{++}$field by a factor of $i$. Therefore, $T$ and $C P$ symmetries are violated only when the matrix $b$ is genuinely complex, neither real nor purely imaginary.

The above results are general and do not rely on any model. It is also possible to preserve lepton number by assigning two units to $\xi^{--}$. Our later calculation of EDM applies to the general case. But since the doubly charged scalars appear naturally in type II seesaw, it is interesting to consider this particular case separately:

$$
b=\frac{1}{2 v_{3}} V^{*} m_{v} V^{\dagger}
$$

where $V$ is the lepton mixing matrix and $m_{v}$ the diagonal neutrino mass matrix with real, semipositive eigenvalues $m_{i}$. The vacuum expectation value of the scalar triplet, $v_{3}$, is induced from that of the scalar doublet through a soft lepton number violating term. It is possible and common practice in type II seesaw to arrange order one Yukawa couplings $b$ by assuming $v_{3}$ to be the same order of magnitude as $m_{v}$. The moduli in $b$ correspond to $n$ neutrino masses (over $\left|v_{3}\right|$ ) plus $\frac{1}{2} n(n-1)$ mixing angles in $V$, while the physical phases are equivalent to $\frac{1}{2}(n-1)(n-2)$ Dirac phases and $(n-1)$ Majorana phases in $V$.

\section{Evaluation of electric dipole moments}

Now we calculate the EDM $d_{\alpha}$ induced for the charged lepton $\ell_{\alpha}$ due to interactions in eqs. (1) 2). The effective EDM interaction is defined as

$$
\mathscr{L}_{\mathrm{EDM}}=-\frac{i}{2} d_{\alpha} \bar{\ell}_{\alpha} \gamma_{5} \sigma_{\mu v} \ell_{\alpha} F^{\mu v}
$$

There is no contribution at one loop level since the matrix element $b_{\alpha \beta}$ always appears in a self-conjugate form, $\left|b_{\alpha \beta}\right|^{2}$, so that no phases can survive. The other way to see this is to notice that, when computing $d_{\alpha}$ for a specific $\alpha$, one can choose suitable phases for the $\ell_{\beta}$ fields so that all of $b_{\alpha \beta}$ are real. Thus more factors of $b$ have to be involved to induce an EDM, and the first contribution occurs at two loop level.

The two-loop Feynman diagrams contributing to $d_{\alpha}$ are depicted in Fig. 1. The incoming and outgoing momenta of the $\ell_{\alpha}$ are respectively $p \pm \frac{1}{2} q$ with $q$ being the outgoing momentum of the photon attached at the vertex indicated by $\otimes$. The arrows in the graphs denote the flow of negative charges, and the summation over the virtual charged leptons $\ell_{\beta}, \ell_{\gamma}$ and $\ell_{\delta}$ is implied. We find that because of the chirality structure in $\mathscr{L}_{\text {Yuk }}$ the chirality flip required by the EDM has to be done by the external lepton mass, $m_{\alpha}$. Upon extracting out this mass factor we ignore further dependence on it. This is a good approximation for practical purposes with incurred

relative errors of order $O\left(r_{\alpha}\right)$, where $r_{\alpha}=m_{\alpha}^{2} / m^{2}$ and $m$ is the mass of $\xi^{ \pm \pm}$. The dependence on other charged lepton masses enters in a quadratic form, i.e., via $r_{\beta, \gamma, \delta}$. 
The lepton flavor dependence in the relevant term of each graph can thus be described as

$$
b_{\gamma \alpha}^{*} b_{\delta \beta}^{*} b_{\delta \gamma} b_{\beta \alpha} f\left(r_{\beta}, r_{\gamma} ; r_{\delta}\right),
$$

where $f$ is a function of the indicated mass ratios. $f$ is generally a sum of terms that are respectively symmetric and antisymmetric in $\beta$ and $\gamma$. The symmetric term cannot contribute to EDM since we are effectively summing the self-conjugated $b$ factors, $b_{\gamma \alpha}^{*} b_{\delta \beta}^{*} b_{\delta \gamma} b_{\beta \alpha}+$ c.c., which do not vanish even for a real or purely imaginary $b$. The antisymmetric combination on the other hand survives only when $b$ is genuinely complex with $\mathrm{CP}$ phases involved:

$$
\frac{i}{2} \mathfrak{J}\left[b_{\gamma \alpha}^{*} b_{\delta \beta}^{*} b_{\delta \gamma} b_{\beta \alpha}\right]\left[f\left(r_{\beta}, r_{\gamma} ; r_{\delta}\right)-f\left(r_{\gamma}, r_{\beta} ; r_{\delta}\right)\right]
$$

Since $r_{\delta} \ll 1$, the leading term, if not vanishing, is obtained by setting $r_{\delta}=0$. The $b$ factors then degenerate into the form

$$
\mathfrak{I}\left[b_{\gamma \alpha}^{*} b_{\beta \alpha}\left(b^{\dagger} b\right)_{\beta \gamma}\right]
$$

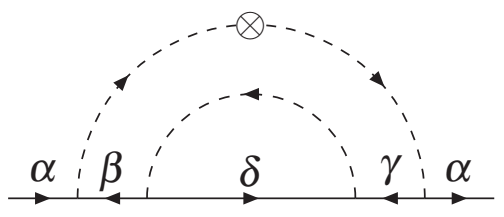

(a)

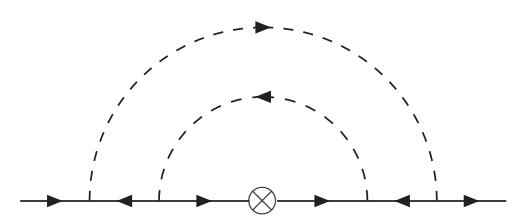

(c)

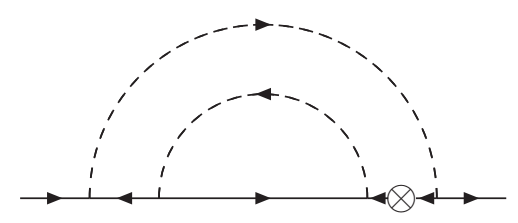

(e)

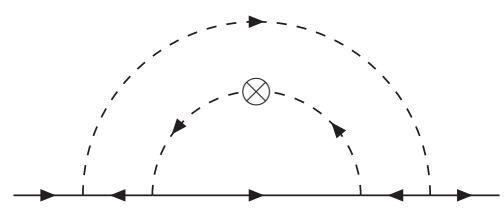

(b)

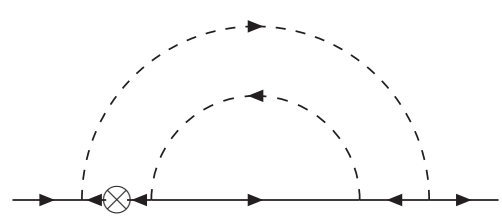

(d)

Figure 1. Diagrams contributing to EDM of $\ell_{\alpha}$.

It is interesting that the above form does not vanish even in the case of two flavors where only a single Majorana phase can appear. To see the point, it suffices to consider the easier case of type II seesaw in eq. (3) with

$$
V=\left(\begin{array}{cc}
c & s \\
-s & c
\end{array}\right)\left(\begin{array}{ll}
u & \\
& 1
\end{array}\right),
$$


where $c, s$ are the cosine and sine of the mixing angle, and $u$ is the $\mathrm{CP}$ phase. Then, we find for instance

$$
\left(2\left|v_{3}\right|\right)^{4} 2 i \mathfrak{I}\left[b_{\mu e}^{*} b_{e e}\left(b^{\dagger} b\right)_{e \mu}\right]=c^{2} s^{2}\left(m_{1}^{2}-m_{2}^{2}\right) m_{1} m_{2}\left(u^{2}-u^{* 2}\right)
$$

which does not vanish in general. This is a feature pertaining to the Majorana-type couplings of charged leptons in eq. (1) or the Majorana nature of neutrinos in type II seesaw.

We will see in the next section that the combination $b_{\delta \beta}^{*} b_{\delta \gamma}$ is no less constrained than $\left(b^{\dagger} b\right)_{\beta \gamma}$. It is thus a good approximation to keep the leading term at $r_{\delta}=0$ while ignoring small corrections that are at most of order $r_{\delta} \ln r_{\delta}$. The final answer for $d_{\alpha}$ thus looks like

$$
d_{\alpha}=C \frac{e m_{\alpha}}{m^{2}} \mathfrak{I}\left[b_{\gamma \alpha}^{*} b_{\beta \alpha}\left(b^{\dagger} b\right)_{\beta \gamma}\right]\left[f\left(r_{\beta}, r_{\gamma} ; 0\right)-f\left(r_{\gamma}, r_{\beta} ; 0\right)\right]
$$

where $C$ is a loop factor. This result entails an interesting sum rule

$$
\frac{d_{e}}{m_{e}}+\frac{d_{\mu}}{m_{\mu}}+\frac{d_{\tau}}{m_{\tau}}=0
$$

which is exact up to small relative corrections of $O\left(r_{\alpha, \delta}\right)$. And up to logarithmic enhancements, we have approximately,

$$
d_{\alpha} \sim C \frac{e m_{\alpha}\left(r_{\beta}-r_{\gamma}\right)}{m^{2}} \mathfrak{I}\left[b_{\gamma \alpha}^{*} b_{\beta \alpha}\left(b^{\dagger} b\right)_{\beta \gamma}\right]
$$

We are now ready to present the results for the graphs. Graph (a) is symmetric in $\beta$ and $\gamma$, and does not contribute to EDM. Graphs (b) and (c) each contain symmetric and antisymmetric terms, while the sum of (d) and (e) is antisymmetric. The contribution to EDM is

$$
d_{\alpha}=\frac{2^{5} e m_{\alpha}}{24(4 \pi)^{4} m^{2}} \mathfrak{I}\left[b_{\gamma \alpha}^{*} b_{\beta \alpha}\left(b^{\dagger} b\right)_{\beta \gamma}\right] J\left(r_{\beta}, r_{\gamma}\right)
$$

where again summation over $\beta, \gamma$ is implied and $J$ is a sum over four graphs:

$$
J\left(r_{\beta}, r_{\gamma}\right)=J^{(b)}\left(r_{\beta}, r_{\gamma}\right)+J^{(c)}\left(r_{\beta}, r_{\gamma}\right)+J^{(d+e)}\left(r_{\beta}, r_{\gamma}\right)
$$

Each of these four graphs has ultraviolet sub-divergences. In $4-2 \varepsilon$ dimensions, they are

$$
J^{(b) \operatorname{div}}=-2 J^{(c) \operatorname{div}}=-2 J^{(d+e) \operatorname{div}}=F\left(r_{\beta}, r_{\gamma}\right) \Gamma(\varepsilon),
$$

where the arguments in $J$ are suppressed and

$$
\begin{aligned}
F(b, c)= & -\frac{2\left[b^{2}+c^{2}-b c-b c(b+c)+b^{2} c^{2}\right]}{(b-1)^{2}(c-1)^{2}(b-c)} \\
& +\frac{b^{2}[-3 c+b(1+b+c)] \ln b}{(b-1)^{3}(b-c)^{2}}-\frac{c^{2}[-3 b+c(1+b+c)] \ln c}{(c-1)^{3}(b-c)^{2}}
\end{aligned}
$$

The divergences are canceled on summation as they must be. 
The analytic result for the finite part is much more lengthy. In addition to the displayed function $F$, each graph involves one or two other twofold parameter integrals that can be worked out in terms of the fractions, logarithms $\ln r_{\beta}$ and $\ln r_{\gamma}$, and the dilogarithms $\operatorname{Li}_{2}\left(1-r_{\beta}\right)$ and $\mathrm{Li}_{2}\left(1-r_{\gamma}\right)$. We will not record these exact results but the sum of all graphs that has been expanded to the leading order in $r_{\beta, \gamma}$ :

$$
J\left(r_{\beta}, r_{\gamma}\right)=\frac{r_{\beta}^{2}+r_{\gamma}^{2}-r_{\beta} r_{\gamma}}{r_{\beta}-r_{\gamma}}+\frac{r_{\beta}^{2}\left(r_{\beta}-3 r_{\gamma}\right) \ln r_{\beta}-r_{\gamma}^{2}\left(r_{\gamma}-3 r_{\beta}\right) \ln r_{\gamma}}{2\left(r_{\beta}-r_{\gamma}\right)^{2}}+\cdots,
$$

where the dots stand for higher order terms in $r_{\beta, \gamma}$. Since the charged lepton masses are hierarchical, further expansion is possible; for $1 \gg r_{\beta} \gg r_{\gamma}$, we have

$$
J\left(r_{\beta}, r_{\gamma}\right)=r_{\beta}-2 r_{\gamma}+\frac{1}{2}\left(r_{\beta}-3 r_{\gamma}\right) \ln r_{\beta}+\cdots
$$

We have tested that the leading terms shown in eq. (16) recover the first three digits of the exact results at $m=200 \mathrm{GeV}$ and are good enough for our later numerical analysis.

\section{Numerical analysis}

Our result for the charged lepton EDMs shown in eqs. (13]16) is suppressed by charged lepton masses squared over four powers of the scalar mass, and has a mild logarithmic enhancement factor. This is a parametrically large contribution. For instance, at our reference point $m=$ $200 \mathrm{GeV}$, we have $J\left(r_{e}, r_{\mu}\right) \approx 1.83 \times 10^{-6}, J\left(r_{e}, r_{\tau}\right) \approx J\left(r_{\mu}, r_{\tau}\right) \approx 2.94 \times 10^{-4}$, and

$$
d_{e} \sim 4 \times 10^{-30} \times[b \text { factors }] e \mathrm{~cm}
$$

which would be within the reach in the next generation of experiment at the sensitivity of order $10^{-31} e \mathrm{~cm}[19]$.

However, the same Yukawa couplings in eq. (1) induce other effects as well, and a realistic estimate of EDM should take into account the constraints from those effects. In this section, we present our numerical results in two approaches. The main constraints considered are from lepton flavor violating (LFV) decays of charged leptons. Also mentioned are anomalous magnetic moments and $0 v 2 \beta$ decays. We start with a model independent analysis in the next subsection and then specialize to the case of type II seesaw. The constraints in the second approach are more stringent because of less free parameters involved.

\subsection{Approach 1: model independent result}

The Yukawa couplings in eq. (1) mediate radiative LFV decays at one loop level and purely leptonic decays at tree level. The branching ratio for the radiative decay is

$$
\operatorname{Br}\left(\ell_{\beta} \rightarrow \ell_{\alpha} \gamma\right)=\frac{3^{3} \alpha}{2^{6} \pi}\left|\frac{\left(b^{\dagger} b\right)_{\alpha \beta}}{G_{F} m^{2}}\right|^{2} B_{\beta} B_{\xi}
$$




\begin{tabular}{|c|l|l|l|l|l|}
\hline modes & $\mu \rightarrow e \gamma$ & $\tau \rightarrow e \gamma$ & $\tau \rightarrow \mu \gamma$ & $\mu \rightarrow 3 e$ & $\tau \rightarrow 3 e$ \\
\hline $\mathrm{Br}$ & $1.210^{-11}[\mathbf{2 0}]$ & $1.110^{-7}[21]$ & $4.510^{-8}[22]$ & $1.010^{-12}[23]$ & $4.310^{-8}[24]$ \\
\hline bounds & $1.210^{-4}$ & $2.910^{-2}$ & $1.910^{-2}$ & $2.010^{-6}$ & $1.010^{-3}$ \\
\hline \hline modes & $\tau \rightarrow 3 \mu$ & $\tau \rightarrow \bar{e} 2 \mu$ & $\tau \rightarrow \bar{\mu} 2 e$ & $\tau \rightarrow \bar{e} e \mu$ & $\tau \rightarrow \bar{\mu} \mu e$ \\
\hline $\mathrm{Br}$ & $5.310^{-8}[24]$ & $5.610^{-8}[24]$ & $5.810^{-8}[24]$ & $8.010^{-8}[24]$ & $3.710^{-8}[24]$ \\
\hline bounds & $1.110^{-3}$ & $1.110^{-3}$ & $1.210^{-3}$ & $9.710^{-4}$ & $6.610^{-4}$ \\
\hline
\end{tabular}

Table 1: Experimental upper bounds on branching ratios of decays in eqs. (19, 20) set upper bounds on $\left|\left(b^{\dagger} b\right)_{\alpha \beta}\right| /\left(G_{F} m^{2}\right)$ and $\left|b_{\delta \alpha} b_{\beta \gamma}\right| /\left(G_{F} m^{2}\right)$ respectively.

with $B_{\mu}=1$ and $B_{\tau} \approx 17 \%$. $B_{\xi}$ is a model parameter which equals $(8 / 9)^{2}$ for the contribution of $\xi^{ \pm \pm}$alone (in this subsection) and equals 1 when both $\xi^{ \pm \pm}$and $\xi^{ \pm}$are included as in type II seesaw model (in the next). The branching ratio for the purely leptonic decay is

$$
\operatorname{Br}\left(\ell_{\delta} \rightarrow \bar{\ell}_{\alpha} \ell_{\beta} \ell_{\gamma}\right)=\frac{1}{2^{2}}\left|\frac{b_{\delta \alpha} b_{\beta \gamma}}{G_{F} m^{2}}\right|^{2}\left(2-\delta_{\beta \gamma}\right) B_{\delta},
$$

which is only induced by $\xi^{ \pm \pm}$exchange. The factor $\left(2-\delta_{\beta \gamma}\right)$ distinguishes between identical and nonidentical particles in the final state. Using the experimental bounds on the branching ratios we can constrain $\left|\left(b^{\dagger} b\right)_{\alpha \beta}\right| /\left(G_{F} m^{2}\right)$ and $\left|b_{\delta \alpha} b_{\beta \gamma}\right| /\left(G_{F} m^{2}\right)$ respectively. These numbers are shown in table 1, and will be employed to set conservative upper bounds on EDMs.

Each $d_{\alpha}$ has three terms proportional to $J\left(r_{e}, r_{\mu}\right), J\left(r_{e}, r_{\tau}\right)$, and $J\left(r_{\mu}, r_{\tau}\right)$ respectively, for instance,

$$
\begin{aligned}
\frac{96 \pi^{4}}{G_{F}^{2}} \frac{d_{e}}{e m_{e}}= & +\mathfrak{I}\left[\frac{b_{\mu e}^{*} b_{e e}}{m^{2} G_{F}} \frac{\left(b^{\dagger} b\right)_{e \mu}}{m^{2} G_{F}}\right] m^{2} J\left(r_{e}, r_{\mu}\right) \\
& +\mathfrak{I}\left[\frac{b_{\tau e}^{*} b_{e e}}{m^{2} G_{F}} \frac{\left(b^{\dagger} b\right)_{e \tau}}{m^{2} G_{F}}\right] m^{2} J\left(r_{e}, r_{\tau}\right) \\
& +\mathfrak{I}\left[\frac{b_{\tau e}^{*} b_{\mu e}}{m^{2} G_{F}} \frac{\left(b^{\dagger} b\right)_{\mu \tau}}{m^{2} G_{F}}\right] m^{2} J\left(r_{\mu}, r_{\tau}\right)
\end{aligned}
$$

The first term is much smaller because of a smaller $J$ factor and more severely suppressed moduli of the products of $b$ factors, and can safely be ignored. In the optimistic case where the products of $b$ factors in the last two terms are purely imaginary and add constructively, we get at $m=200 \mathrm{GeV}$,

$$
\left|d_{e}\right| \leq 8.1 \times 10^{-35} e \mathrm{~cm}
$$

Since our bounds in table 1 are given independently of $m^{2}$ while $m^{2} J$ depends only logarithmically on $m^{2}$, the above bound is stable against mild variations of $m$. Similarly, we obtain

$$
\left|d_{\mu}\right| \leq 1.4 \times 10^{-32} \text { e cm }
$$


The expression for $d_{\tau}$ contains several combinations of $b$ factors that cannot be constrained in LFV decays, so that a direct bound is not possible. But we can utilize the sum rule (11) to set a bound

$$
\left|d_{\tau}\right| \leq 5.2 \times 10^{-31} e \mathrm{~cm}
$$

The limit on $\left|d_{e}\right|$, though larger than the four-loop SM result [12] and the bounds reached via other mechanisms [14, 15, 16, 17], is still about three orders of magnitude below the precision reachable in the near future [19].

\subsection{Approach 2: a case study in type II seesaw}

The discussion in the previous subsection is model independent. When the Yukawa interaction in eq. (1) is part of a complete structure in a model, more stringent constraints on EDMs can be obtained. This is the case particularly in the type II seesaw model where the Yukawa couplings are related via eq. (3) to the neutrino masses and mixing matrix which have been determined to certain extent. In this subsection we will not attempt a global fitting but demonstrate the point by a case study in this model.

The mixing pattern determined by oscillation data is close to the tribimaximal texture [25]. We will work in this simplified scenario. There is then no Dirac phase but there can be two Majorana phases $u_{1,2}$ :

$$
V=\left(\begin{array}{ccc}
\sqrt{\frac{2}{3}} u_{1} & \frac{1}{\sqrt{3}} u_{2} & 0 \\
-\frac{1}{\sqrt{6}} u_{1} & \frac{1}{\sqrt{3}} u_{2} & \frac{1}{\sqrt{2}} \\
\frac{1}{\sqrt{6}} u_{1} & -\frac{1}{\sqrt{3}} u_{2} & \frac{1}{\sqrt{2}}
\end{array}\right)
$$

Then, the matrix $b^{\dagger} b$ is real and symmetric,

$$
\begin{aligned}
4\left|v_{3}\right|^{2} b^{\dagger} b= & \frac{1}{3}\left(m_{1}^{2}+m_{2}^{2}+m_{3}^{2}\right) 1_{3} \\
& +\frac{1}{6}\left(\begin{array}{ccc}
2 \Delta_{13} & -2 \Delta_{12} & 2 \Delta_{12} \\
-2 \Delta_{12} & -\Delta_{13} & -\Delta_{13}-2 \Delta_{23} \\
2 \Delta_{12} & -\Delta_{13}-2 \Delta_{23} & -\Delta_{13}
\end{array}\right)
\end{aligned}
$$

with $\Delta_{i j}=m_{i}^{2}-m_{j}^{2}$.

The off-diagonal moduli $\left|\left(b^{\dagger} b\right)_{\alpha \beta}\right|$ depend explicitly on $\Delta_{i j}$, which have been determined e.g. in [26] to be, $\Delta_{21}=7.6 \times 10^{-5} \mathrm{eV}^{2},\left|\Delta_{31}\right|=2.4 \times 10^{-3} \mathrm{eV}^{2}$. The bound on $\operatorname{Br}(\mu \rightarrow e \gamma)$ then implies (using $B_{\xi}=1$ )

$$
\left|v_{3}\right|^{2} m^{2} G_{F}>5.75 \times 10^{-2} \mathrm{eV}^{2}
$$

Since $\operatorname{Br}(\tau \rightarrow e \gamma)$ also depends on $\Delta_{12}$, its less stringent bound is useless. Instead, its relation to $\operatorname{Br}(\mu \rightarrow e \gamma)$ in type II seesaw and the experimental bound on the latter mean

$$
\operatorname{Br}(\tau \rightarrow e \gamma)=B_{\tau} \operatorname{Br}(\mu \rightarrow e \gamma) \leq 2.0 \times 10^{-12}
$$


which is much below the current bound. We also notice that the bound on $\operatorname{Br}(\tau \rightarrow \mu \gamma)$, though more than three orders of magnitude larger than $\operatorname{Br}(\mu \rightarrow e \gamma)$, gives a constraint that is only slightly weaker than in eq. (27), because of an enhancement factor $\left|\Delta_{31}\right| / \Delta_{21}$. A similar relation also holds between $\operatorname{Br}(\tau \rightarrow 3 e)$ and $\operatorname{Br}(\mu \rightarrow 3 e)$; the more stringent bound on the latter implies

$$
\operatorname{Br}(\tau \rightarrow 3 e)=B_{\tau} \operatorname{Br}(\mu \rightarrow 3 e) \leq 1.7 \times 10^{-13}
$$

which is much smaller than its current bound and thus more difficult to observe than other decay modes of $\tau$.

To proceed further with leptonic decays of $\tau$ and EDM, we set all neutrino masses in $b_{\delta \alpha}^{*} b_{\beta \gamma}$ (but not in $\left(b^{\dagger} b\right)_{\beta \alpha}$ of course) to be equal to their average value $\bar{m}_{v}$. This simplification holds true barring very delicate cancellation among neutrino mass differences and Majorana phases. Then, the most stringent constraints on $\mu \rightarrow 3 e, \tau \rightarrow \bar{e} e \mu, \bar{\mu} \mu e$ (together with the less stringent one on $\tau \rightarrow \bar{e} 2 \mu$ ) are proportional to $\left|u_{1}^{2}-u_{2}^{2}\right|$. We may reach the most optimistic values of EDMs by assuming $u_{1}^{2}=u_{2}^{2}=e^{i \phi}$ to avoid these bounds. The remaining ones on $\tau \rightarrow 3 \mu, \bar{\mu} 2 e$ yield comparable constraints:

$$
\frac{\bar{m}_{v}^{2}|\sin \phi|}{8\left|v_{3}\right|^{2} m^{2} G_{F}}<1.1 \times 10^{-3}, \frac{\bar{m}_{v}^{2}|\sin (\phi / 2)|}{4\left|v_{3}\right|^{2} m^{2} G_{F}}<1.2 \times 10^{-3}
$$

The electron EDM being proportional to $\mathfrak{I}\left(u_{1}^{* 2} u_{2}^{2}\right)$ vanishes, while the other two simplify to

$$
\frac{d_{\mu}}{e m_{\mu}}=-\frac{d_{\tau}}{e m_{\tau}}=\frac{\bar{m}_{v}^{2} \Delta_{13} \sin \phi}{2^{11} 3 \pi^{4}\left|v_{3}\right|^{4} m^{2}} J\left(r_{\mu}, r_{\tau}\right)
$$

barring cancellation of $O\left(\Delta_{21} /\left|\Delta_{31}\right|\right) \sim 3 \%$ or $O\left(\left[\Delta_{21} J\left(r_{e}, r_{\mu}\right)\right] /\left[\left|\Delta_{31}\right| J\left(r_{\mu}, r_{\tau}\right)\right]\right) \sim 2 \times 10^{-4}$. The bounds in eqs. (27, 30) then give at $m=200 \mathrm{GeV}$

$$
\left|d_{\mu}\right|<2.0 \times 10^{-33} e \mathrm{~cm},\left|d_{\tau}\right|<3.4 \times 10^{-32} e \mathrm{~cm}
$$

As expected, this result is better than the model-independent one in the previous subsection.

In this special scenario, the effective neutrino mass measured in $0 v 2 \beta$ decays is $m_{\beta \beta}=$ $\left(2 m_{1}+m_{2}\right) / 3 \sim \bar{m}_{v}$. The contributions to anomalous magnetic dipole moments depend on the diagonal elements of $b^{\dagger} b$ and are given by

$$
\begin{aligned}
a_{e} & =\frac{3}{(4 \pi)^{2}} \frac{\bar{m}_{v}^{2} m_{e}^{2} G_{F}}{4\left|v_{3}\right|^{2} m^{2} G_{F}}<1.1 \times 10^{-14} \\
a_{\mu} & =\frac{3}{(4 \pi)^{2}} \frac{\bar{m}_{v}^{2} m_{\mu}^{2} G_{F}}{4\left|v_{3}\right|^{2} m^{2} G_{F}}<4.7 \times 10^{-10}
\end{aligned}
$$

using eq. 27) and $\bar{m}_{V} \sim 0.21 \mathrm{eV}$ from a recent update of cosmological bounds on the sum of neutrino masses [27]. Both $a_{e}$ and $a_{\mu}$ are below the potential gap between measurements and SM expectations [28, 29]. 


\section{Conclusion}

$\mathrm{CP}$ violation in the lepton sector has remained an experimentally unexplored issue. The charged lepton EDMs offer a potential arena to detect it. This is especially encouraged by the experimental precision in EDM measurements that has been reached and will possibly be accessible. However, it has been found previously that it is hard to obtain a not too tiny EDM for charged leptons from a flavor CP source. This may be blamed on the very light, thus almost degenerate at the electroweak scale, neutrinos. Together with a small mixing angle out of three, this makes a Dirac phase effectively unobservable; and it suppresses the effects of Majorana phases on EDMs by several factors of neutrino masses. We have thus been motivated to consider a CP source that arises from Majorana-type Yukawa couplings of charged leptons. Such couplings may appear naturally in SM with an extended scalar sector, such as type II seesaw model, but we have presented our analytic results in a general setting. We found that the EDMs so obtained are parametrically large. They are only suppressed by charged lepton masses squared over four powers of heavy scalar masses for order one Yukawa couplings that may be naturally arranged, for instance, in type II seesaw model by assigning a tiny vacuum expectation value for the scalar triplet.

Nevertheless, the fate with a flavor CP source seems insurmountable. While a large enough EDM, though flavor diagonal, demands reasonably large flavor changing couplings, this may not be allowed by strictly bounded LFV transitions. With these bounds taken into account, we found that the electron EDM is at least three orders of magnitude below the precision achievable in the near future, although it is still much larger than the contributions considered previously.

Acknowledgement This work is supported in part by the grants NCET-06-0211 and NSFC10775074.

\section{References}

[1] M. Fukugita and T. Yanagida, Phys. Lett. B 174, 45 (1986).

[2] V. A. Kuzmin, V. A. Rubakov and M. E. Shaposhnikov, Phys. Lett. B 155, 36 (1985).

[3] For reviews, see e.g.: M. Maltoni and T. Schwetz, Phys. Rev. D 76, 093005 (2007) [arXiv:0705.0107 [hep-ph]]; A. Strumia and F. Vissani, arXiv:hep-ph/0606054; Z. z. Xing, Int. J. Mod. Phys. A 19, 1 (2004) [arXiv:hep-ph/0307359].

[4] M. V. Romalis, W. C. Griffith and E. N. Fortson, Phys. Rev. Lett. 86, 2505 (2001) [arXiv:hep-ex/0012001].

[5] C. A. Baker et al., Phys. Rev. Lett. 97, 131801 (2006) [arXiv:hep-ex/0602020]. 
[6] B. C. Regan, E. D. Commins, C. J. Schmidt and D. DeMille, Phys. Rev. Lett. 88, 071805 (2002).

[7] G. W. Bennett et al., arXiv:0811.1207 [hep-ex].

[8] For a comprehensive review, see e.g., M. Pospelov and A. Ritz, Annals Phys. 318, 119 (2005) [arXiv:hep-ph/0504231].

[9] E. P. Shabalin, Sov. J. Nucl. Phys. 28, 75 (1978) [Yad. Fiz. 28, 151 (1978)].

[10] Y. Liao and X. y. Li, Phys. Rev. D 60, 073004 (1999) [arXiv:hep-ph/9902473].

[11] Y. Liao and X. y. Li, Phys. Rev. D 61, 076002 (2000) [arXiv:hep-ph/9906245]; Y. Liao and X. Li, Phys. Lett. B 503, 301 (2001) [arXiv:hep-ph/0005063].

[12] M. E. Pospelov and I. B. Khriplovich, Sov. J. Nucl. Phys. 53, 638 (1991) [Yad. Fiz. 53, 1030 (1991)].

[13] For an interesting illustration on peculiarities of Majorana CP phases, see e.g., A. de Gouvea, B. Kayser and R. N. Mohapatra, Phys. Rev. D 67, 053004 (2003) [arXiv:hep-ph/0211394].

[14] D. Ng and J. N. Ng, Mod. Phys. Lett. A 11, 211 (1996) [arXiv:hep-ph/9510306].

[15] J. P. Archambault, A. Czarnecki and M. Pospelov, Phys. Rev. D 70, 073006 (2004) [arXiv:hep-ph/0406089].

[16] W. F. Chang and J. N. Ng, New J. Phys. 7, 65 (2005) [arXiv:hep-ph/0411201].

[17] A. de Gouvea and S. Gopalakrishna, Phys. Rev. D 72, 093008 (2005) [arXiv:hep-ph/0508148].

[18] W. Konetschny and W. Kummer, Phys. Lett. B 70, 433 (1977); T. P. Cheng and L. F. Li, Phys. Rev. D 22, 2860 (1980); J. Schechter and J. W. F. Valle, Phys. Rev. D 22, 2227 (1980).

[19] D. Kawall, F. Bay, S. Bickman, Y. Jiang and D. DeMille, AIP Conf. Proc. 698, 192 (2004).

[20] M. L. Brooks et al. [MEGA Collaboration], Phys. Rev. Lett. 83, 1521 (1999) [arXiv:hep-ex/9905013].

[21] B. Aubert et al. [BABAR Collaboration], Phys. Rev. Lett. 96, 041801 (2006) [arXiv:hep-ex/0508012].

[22] K. Hayasaka et al. [Belle Collaboration], Phys. Lett. B 666, 16 (2008) [arXiv:0705.0650 [hep-ex]]. 
[23] U. Bellgardt et al. [SINDRUM Collaboration], Nucl. Phys. B 299, 1 (1988).

[24] B. Aubert et al. [BABAR Collaboration], Phys. Rev. Lett. 99, 251803 (2007) [arXiv:0708.3650 [hep-ex]].

[25] P. F. Harrison, D. H. Perkins and W. G. Scott, Phys. Lett. B 530, 167 (2002) [arXiv:hep-ph/0202074]; Z. z. Xing, Phys. Lett. B 533, 85 (2002) [arXiv:hep-ph/0204049]; P. F. Harrison and W. G. Scott, Phys. Lett. B 535, 163 (2002) arXiv:hep-ph/0203209]; X. G. He and A. Zee, Phys. Lett. B 560, 87 (2003) [arXiv:hep-ph/0301092].

[26] J. Garayoa and T. Schwetz, JHEP 0803, 009 (2008) [arXiv:0712.1453 [hep-ph]].

[27] S. Hannestad, A. Mirizzi, G. G. Raffelt and Y. Y. Y. Wong, JCAP 0804, 019 (2008) [arXiv:0803.1585 [astro-ph]].

[28] B. C. Odom, D. Hanneke, B. D’Urso and G. Gabrielse, Phys. Rev. Lett. 97, 030801 (2006) [Erratum-ibid. 99, 039902 (2007)].

[29] W. M. Yao et al. [Particle Data Group], J. Phys. G 33, 1 (2006). 